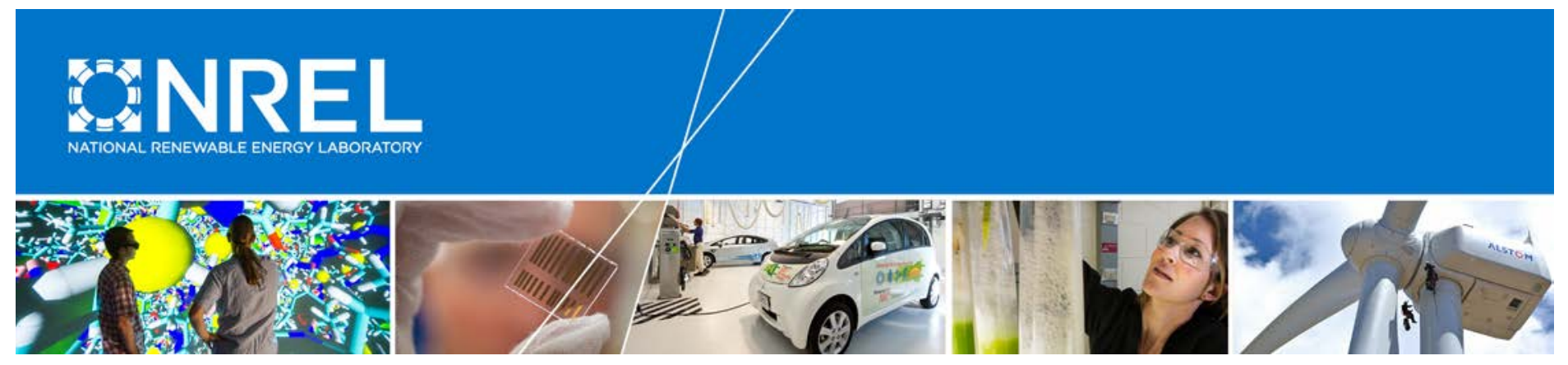

\title{
The Trip Itinerary Optimization Platform: A Framework for Personalized Travel Information
}

Ted Kwasnik, Steve Isley, Scott P. Carmichael, Joshua B. Sperling, and Doug Arent National Renewable Energy Laboratory

NREL is a national laboratory of the U.S. Department of Energy Office of Energy Efficiency \& Renewable Energy Operated by the Alliance for Sustainable Energy, LLC

This report is available at no cost from the National Renewable Energy Laboratory (NREL) at www.nrel.gov/publications.

Technical Report

NREL/TP-6A80-67241

November 2017 


\section{The Trip Itinerary Optimization Platform: A Framework for Personalized Travel Information}

Ted Kwasnik, Steve Isley, Scott P. Carmichael, Joshua B. Sperling, and Doug Arent

National Renewable Energy Laboratory

Prepared under Task No. 0600.10015.14.01.01

NREL is a national laboratory of the U.S. Department of Energy Office of Energy Efficiency \& Renewable Energy Operated by the Alliance for Sustainable Energy, LLC

This report is available at no cost from the National Renewable Energy Laboratory (NREL) at www.nrel.gov/publications.

National Renewable Energy Laboratory 15013 Denver West Parkway Golden, CO 80401 303-275-3000 • www.nrel.gov

\section{Technical Report}

NREL/TP-6A80-67241

November 2017

Contract No. DE-AC36-08G028308 


\title{
NOTICE
}

This report was prepared as an account of work sponsored by an agency of the United States government. Neither the United States government nor any agency thereof, nor any of their employees, makes any warranty, express or implied, or assumes any legal liability or responsibility for the accuracy, completeness, or usefulness of any information, apparatus, product, or process disclosed, or represents that its use would not infringe privately owned rights. Reference herein to any specific commercial product, process, or service by trade name, trademark, manufacturer, or otherwise does not necessarily constitute or imply its endorsement, recommendation, or favoring by the United States government or any agency thereof. The views and opinions of authors expressed herein do not necessarily state or reflect those of the United States government or any agency thereof.

This report is available at no cost from the National Renewable Energy Laboratory (NREL) at www.nrel.gov/publications.

Available electronically at SciTech Connect http:/www.osti.gov/scitech

Available for a processing fee to U.S. Department of Energy and its contractors, in paper, from:

\author{
U.S. Department of Energy \\ Office of Scientific and Technical Information \\ P.O. Box 62 \\ Oak Ridge, TN 37831-0062 \\ OSTI http://www.osti.gov \\ Phone: 865.576.8401 \\ Fax: 865.576.5728 \\ Email: reports@osti.gov
}

Available for sale to the public, in paper, from:

\author{
U.S. Department of Commerce \\ National Technical Information Service \\ 5301 Shawnee Road \\ Alexandria, VA 22312 \\ NTIS http://www.ntis.gov \\ Phone: 800.553 .6847 or 703.605 .6000 \\ Fax: 703.605.6900 \\ Email: orders@ntis.gov
}




\section{Foreword}

Connected and automated information services in coordination with ubiqutious sensing make possible energy systems whose operation, maintenance and future design is informed by continuous feedback from large numbers of individuals collected over long periods of time. As an energy-intensive sector, transportation is well poised to become more human-focused and energy efficient through integration with advanced information technologies.

This study is a preliminary study to explore how automated personal travel assistants can be used to understand what motivates current travel behaviors, as well as what efficiciency gains are feasible through alternative mode choices. The study originated in the New Concept Incubator at NREL, a multidisciplinary research team focused on innovation that simultaneously achieves individual quality of life improvements and large-scale societal gains. 


\section{Acknowledgments}

The authors would like to thank Dr. Stuart MacMillan for his insights and encouragement, as well as Stan Young, Ph.D. and Eric Wood for their thoughtful feedback. We would also like to acknowledge Michael Mehrtens and William Gillies for their contributions to the design and analysis of this study. 


\section{Nomenclature or List of Acronyms}

AHP

AMT

API

AWS

BMR

HIT

MET

NREL
Analytic Hierarchy Process

Amazon Mechanical Turk

Application Programming Interface

Amazon Web Services

Basal Metabolic Rates

Human Intelligence Task

Metabolic Equivalent

National Renewable Energy Laboratory 


\section{Executive Summary}

Optimizations of personal travel plans for a single day can be challenging computations given the dynamic performance of transportation systems, proliferation of mode options and mobility service providers, as well as the diversity of individual travel preferences. Accordingly, the New Concepts Incubator team at the National Renewable Energy Laboratory (NREL) developed the Trip Itinerary Optimization (TrIO) platform to optimize trip itineraries in accordance with the realistic daily constraints and unique values of individual travelers. Over 270 participants used this three-staged web tool. Their responses yield valuable insights into the ways in which individual travel patterns vary from optimal outcomes and how individuals respond to personalized information.

In the first stage, users nationwide that were recruited from the Amazon Mechanical Turk online survey platform provided a travel diary for the previous day. They also responded to a survey about travel attitudes and behaviors. One or more days later, these same users were invited to view a personal mobility dashboard displaying a series of recommended travel itineraries. A typical itinerary may include a trip from home to work, trips to and from a lunch destination, and a final return trip home. Following, each trip's set of feasible modes is subject to time limitations (based on factors such as upcoming calendar events or traffic conditions), possible travel constraints (such as the need to transport passengers or luggage), and the interdependencies among previous and upcoming trips. The travel itineraries recommended to users overcame such logistical considerations through implementation of a novel algorithmic framework, described in this report. The procedure enumerates all feasible combinations of travel mode options and distills optimal outcomes in terms of time, cost, calories burned, carbon dioxide emissions or personal preference. A week or more after viewing recommended travel itineraries on the web dashboard, users were again invited to engage in a final survey about travel attitudes and behaviors.

A preliminary usability study of the platform showed that, in general, users found the system valuable for informing their travel decisions. In fact, $13 \%$ of platform users who viewed the dashboard reported subsequent changes to their travel behavior, most implementing the time, calories or carbon optimized itineraries. On average, the platform distilled 65 feasible travel plans per individual into two recommended itineraries, each optimal according to one or more outcomes and dependent on the fixed times and locations from the travel diary. For slightly less than half of viewers, the trip recommendation algorithm found no viable alternative transportation modes available. This finding is interesting considering about $85 \%$ of those who viewed the dashboard completed two or more trips within urban areas (as defined by the U.S. Census in 2015) where there are typically higher levels public transit infrastructure than rural areas. Platform users generally agreed that the dashboard was enjoyable and easy to use, and that it would be a helpful tool in adopting new travel behaviors. Users generally agreed that the time, cost and user preferred recommendations 'made sense' to them, and were most willing to implement these itineraries. Platform users typically expressed low willingness to try the carbon and calories optimized itineraries. 
The Trip Itinerary Optimization (TrIO) platform incorporates historic traffic condition data, public transit timetables, bike path routes, and other pertinent travel information available via web API's. In open-ended responses, however, users commonly expressed interest in even more fine-grained traffic data and the ability to dynamically model the effect of changes in travel times. Users were also generally concerned over the safety of walking and biking recommendations. Responses indicate that more information about the amenities available to cyclists and pedestrians (sidewalks, shade from trees, access to food) and routes that avoid areas of perceived elevated danger would reduce barriers to implementing such recommended modes.

Further research will also seek to incorporate more fine-grained information about current travel behavior (vehicle make and model, time and money spent parking) to enhance the accuracy and personalization of recommendations. Additionally, the identification of routes that optimize caloric intensity (seeking out elevation changes or longer walks to public transit hubs) will enhance the benefits associated with such recommendations, and possibly lead to higher recommendation adoption rates.

In the context of shared mobility services and other emerging technical innovations, personal mobility applications like the one proposed in this report will play increasingly important role for those living in urban environments. By exposing personalized costs and benefits of multiple relevant alternatives, travelers will be better prepared to adapt their travel behaviors and to achieve personal goals, such as shorter commutes, personal health gains and reduced carbon footprints. Such tools, moreover, provide a platform for those managing transportation networks to take actions and deliver relevant incentives that improve system performance in aggregate. Future development of the Trip Itinerary Optimization (TrIO) platform will provide insights into effective design and incentive strategies for leveraging human-centered information systems alongside existing infrastructure to achieve quality of life improvements and the reduction of adverse environmental impacts. 


\section{Table of Contents}

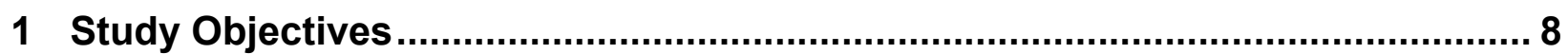

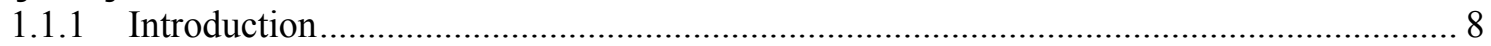

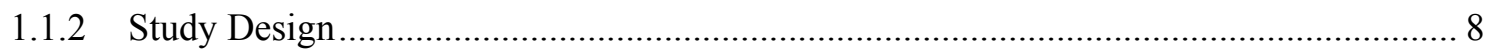

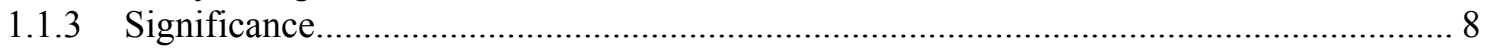

2 Trip Itinerary Optimization (TrIO) Platform .......................................................... 9

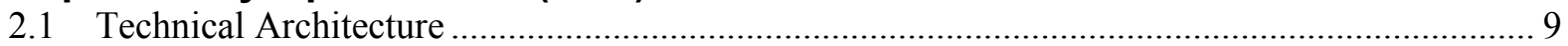

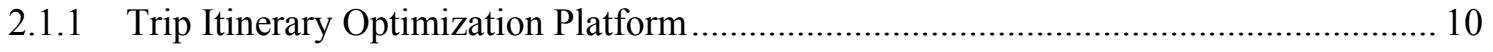

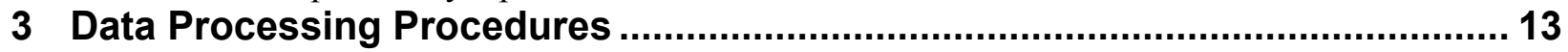

3.1 Task Scheduling ......................................................................................................... 13

3.2 Trip Generation Algorithm....................................................................................... 14

3.2.1 Algorithm Vocabulary ......................................................................................... 14

3.2.2 Algorithm Data Management................................................................................ 17

3.2.3 Recommendation Generation.............................................................................. 18

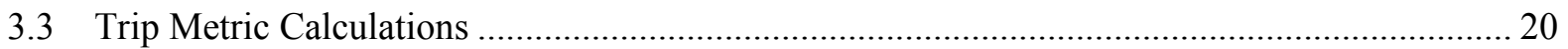

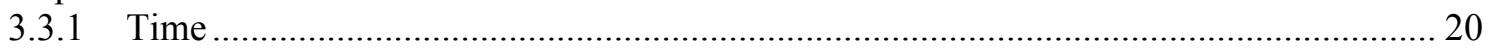

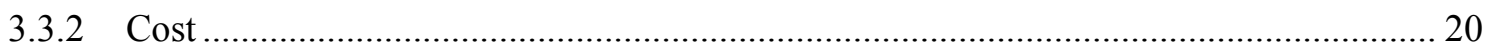

3.3.3 Carbon Emissions .......................................................................................... 21

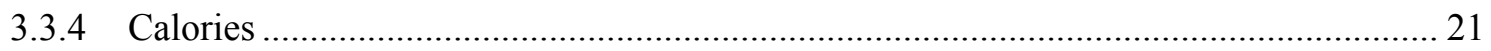

3.3.5 User Preference Score ....................................................................................... 22

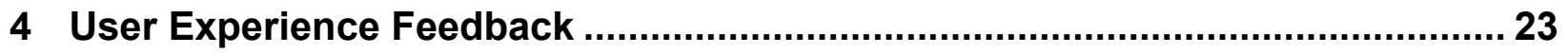

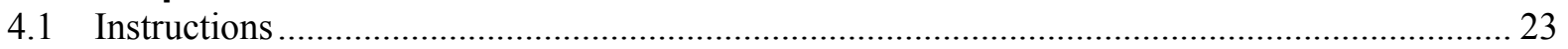

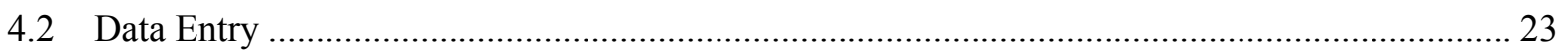

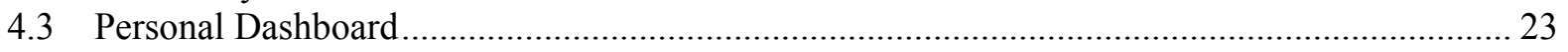

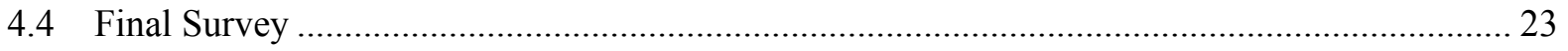

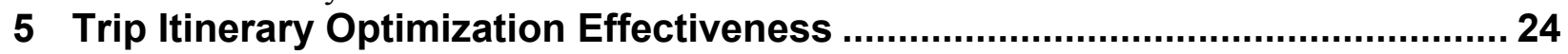

5.1 Simplification of Travel Possibilities ……………………................................................ 24

5.2 Response to Recommended Itineraries ………………………………………………….... 24

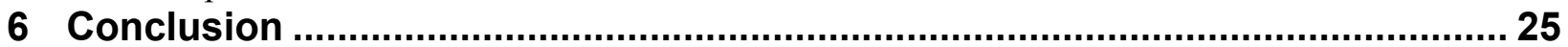

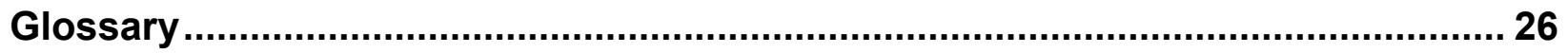

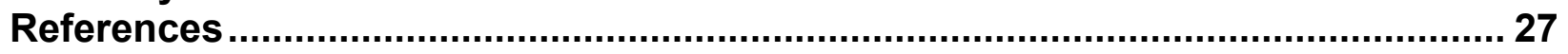

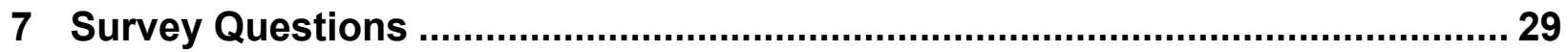

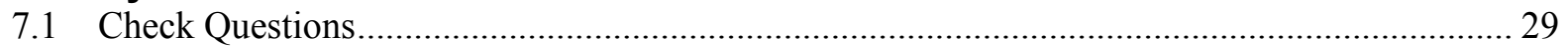

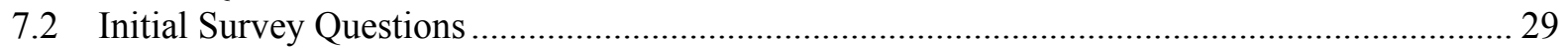

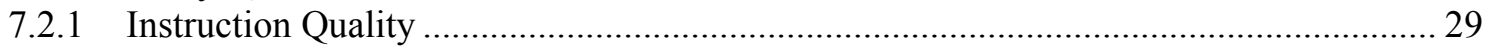

7.2.2 Travel Attitudes and Behaviors............................................................................... 29

7.2.3 Travel Preferences and Constraints......................................................................... 31

7.2.4 Data Entry Experience ……………………………….................................... 32

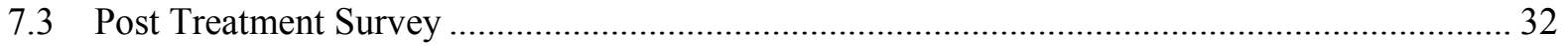

7.3.1 Dashboard Feedback ………………………………………………………... 32

7.3.2 Travel Attitudes and Behaviors............................................................................. 33

7.3.3 Dashboard Design Feedback...……………………….............................................. 33

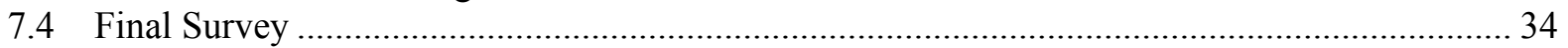

7.4.1 Behavior Change................................................................................................ 34

7.4.2 Travel Attitudes and Behaviors................................................................................ 35

8 Amazon Mechanical Turk (AMT) Platform Specifications ................................. 36

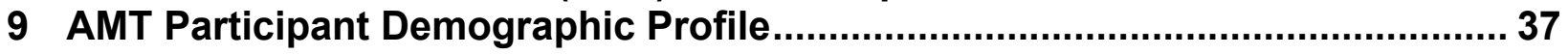

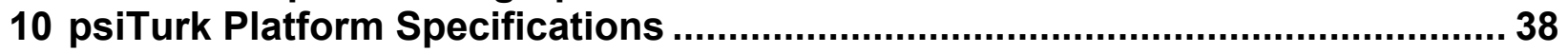




\section{List of Figures}

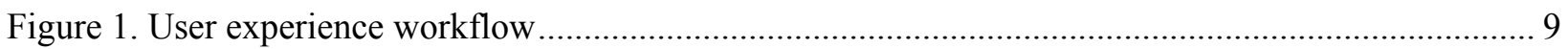

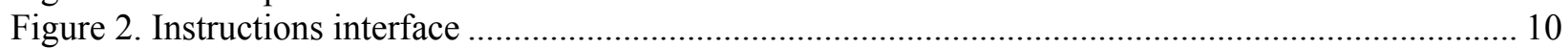

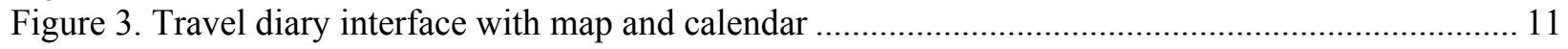

Figure 4. Personal travel dashboard showing a summary of all itineraries............................................. 12

Figure 5. Personal travel dashboard showing an itinerary optimized for user preference ........................ 13

Figure 6. Relationships between trips, trip alternatives, and sections............................................... 15

Figure 7. Relationships between parent and child loops................................................................... 16

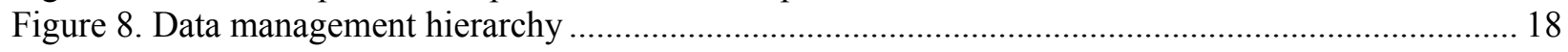

Figure 9. Possible travel scenarios based for one pair of parent-child loops .......................................... 19

\section{List of Tables}

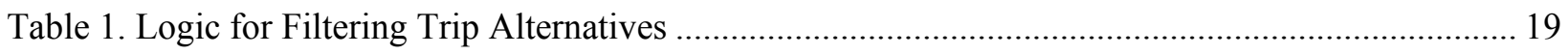

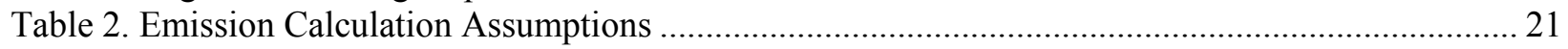

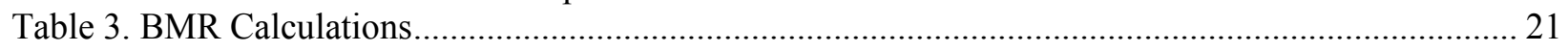

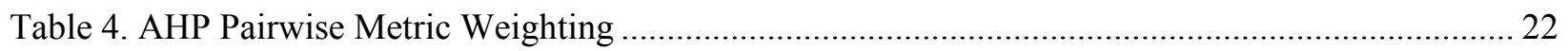

Table 5. Reponses to Recommended Itineraries while Viewing Dashboard .......................................... 24

Table 6. Participant Demographics Throughout Study Phases .............................................................. 37 


\section{Study Objectives}

\subsubsection{Introduction}

Within many urban areas, usage of auto-oriented transportation networks increasingly impeding the system's ability to efficiently transport people and goods (Fujii and Taniguchi 2006; Meloni et al. 2014). Moreover, vehicle emissions and traffic congestion negatively impact human and environmental health through their contributions to productivity losses, air pollution, and climate change (Anagnostopoulou et al. 2016; Binsead et al. 2011; Meloni et al. 2014; Fuji and Taniguchi 2006). For certain situations within urban areas, adoption of alternative modes of transportation including walking, biking, public transit and shared mobility services (including Uber, Lyft, Car2Go) has the potential to mitigate such negative travel outcomes, and also to provide otherwise unrealized benefits to travelers.

Several cognitive barriers exist that limit the wide-scale adoption of optimal transportation modes. Logically, travelers must be aware of alternatives (including emerging shared mobility services) and understand the advantages that they yield before they will voluntarily integrate new modes into their daily travel routines. Additionally, the analysis of tradeoffs between personal travel mode options can be obfuscated by the temporal and geographic variability of transportation system performance and accessibility. Accordingly, information technology aids that reduce disparate travel data resources into actionable information have a potentially important role to play in transportation system optimization.

\subsubsection{Study Design}

Participation in this study was conducted entirely through web-based platforms capable of engaging participants nationwide. Connected and automated information technologies were employed to collect travel diary data, perform optimization analysis, distill personalized travel itineraries, and provide feedback to individuals through an interactive decision support platform.

The online mobility recommendation process consists of three stages spread across several days. Users first provide origin-destination pairs, calendar events and travel constraints (the need to transport passengers or children, baggage, etc.) through an online travel diary interface. Additionally, a preference elicitation survey is given that measured prioritization of optimal time, cost, calories, and carbon emission outcomes when making travel decisions. The travel itinerary details informs a recommendation engine that schedules requests to location-based web services for information including i) traffic conditions, ii) estimated drive times between origindestination pairs, and iii) the cost and availability of public transit options as well as shared mobility services. One or more days later, after feasible travel itineraries have been compiled from these web-based information services, platform users are provided access to a personalized online dashboard displaying sets of daily trip itineraries, each optimized for either time, cost, calories, carbon emissions or user preference. Finally, a week later, users are invited to respond to a final survey about their actual travel behaviors. At all stages, users responded to survey questions designed to quantify travel attitudes and behaviors.

\subsubsection{Significance}

This study proposes a framework for identifying optimal daily travel itineraries for a series of origin-destination pairs. The framework is designed to tailor itineraries to personal travel preferences and real-world constraints. Moreover, it implements mechanisms to account for the 
interaction effects that any single trip may have within a larger chain of trips. The framework is adaptable to the inclusion of additional modes and capable of integrating supplemental travel information from diverse travel data repositories.

\section{Trip Itinerary Optimization (TrIO) Platform}

This section will describe the technical components of managing user engagement throughout the study.

\subsection{Technical Architecture}

Figure 1 illustrates user progression throughout the three stages of this study and the technical environments that supported each step (including Amazon Mechanical Turk, psiTurk, and the Trip Itinerary Optimization (TrIO) platform). For descriptions for our specific implementation of Amazon Mechanical Turk and psiTurk software, as well as more information about the demographics of those who used the platform, refer to Sections 8, 9 and 10.

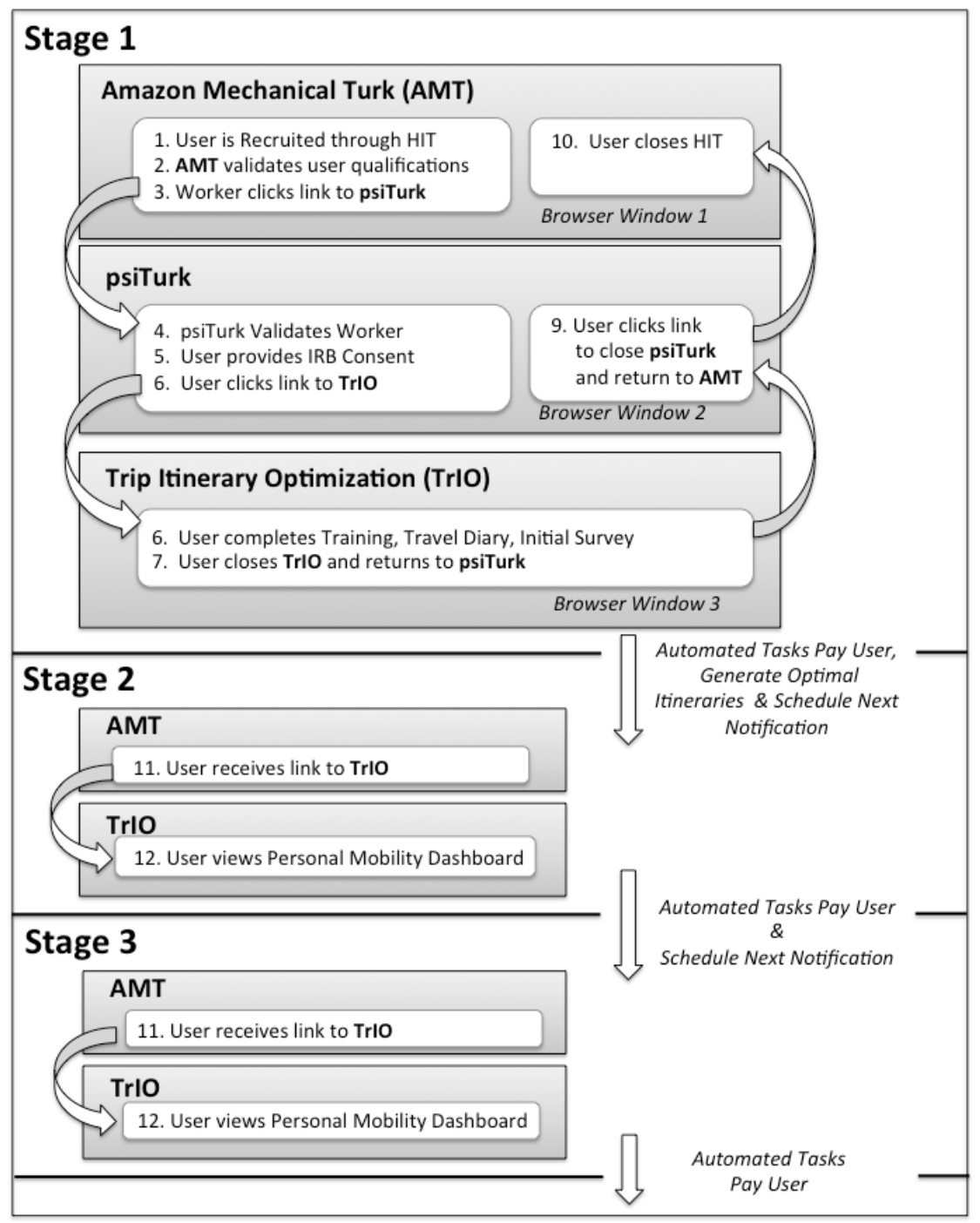

Figure 1. User experience workflow 


\subsubsection{Trip Itinerary Optimization Platform}

The Trip Itinerary Optimization (TrIO) Platform is an online tool for collecting and disseminating travel information. It was hosted on a 2 .small Amazon EC2 instance running the Ubuntu operating system. Web traffic was managed through Python-based server software (Twisted Matrix Labs 2016) and the HTTPS protocol. Each stage of the user's progression through this platform will now be described.

\section{Training}

A tutorial on the travel diary input process introduced users to the platform. In total, the tutorial guided the user through eleven steps that broke out the various stages on entering data for an example day and also provided additional helpful hints. Figure 2 depicts the first training page presented to a user. User feedback suggests that a short training video would be more helpful than the series of static images for training purposes.

\section{Instructions}
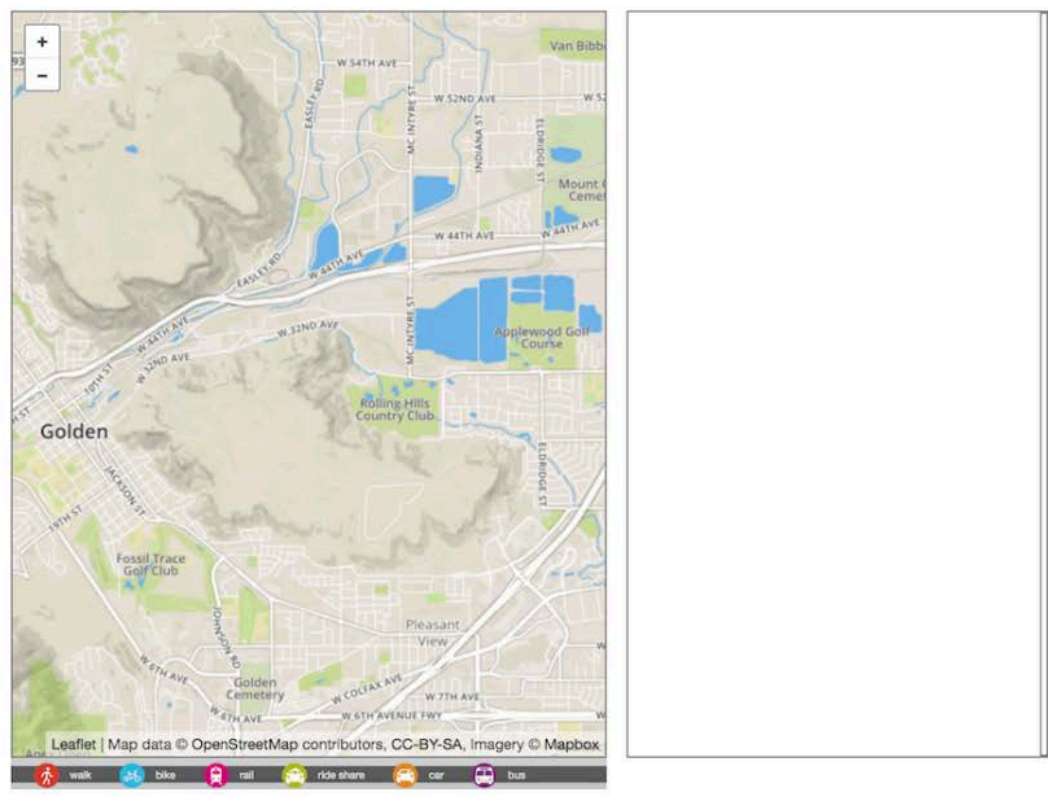

3 of 12

Figure 2. Instructions interface

\section{Check Questions}

For the purpose of the study, check questions were included to ensure platform users read and understood the instructions. After the Travel Diary training, three such questions were asked to check the user attentiveness to the training instructions (See Section 7.1). User responses were accepted in many cases even with incorrect responses to one of these questions. The check questions were consulted when reviewing travel diaries to decide whether to follow up with respondents for information about spurious data entries. 


\section{Travel Diary Data Entry}

Through an interactive travel diary (Figure 3), the user recorded the travel times, destinations, and travel modes for a typical day's travel. As users clicked on the map, moveable destinations markers and connecting trips appeared in the map and on the calendar. Markers were labeled by a name and trips color-coded according to mode. Snapping coerced a common latitude, longitude and name for overlapping destination markers.
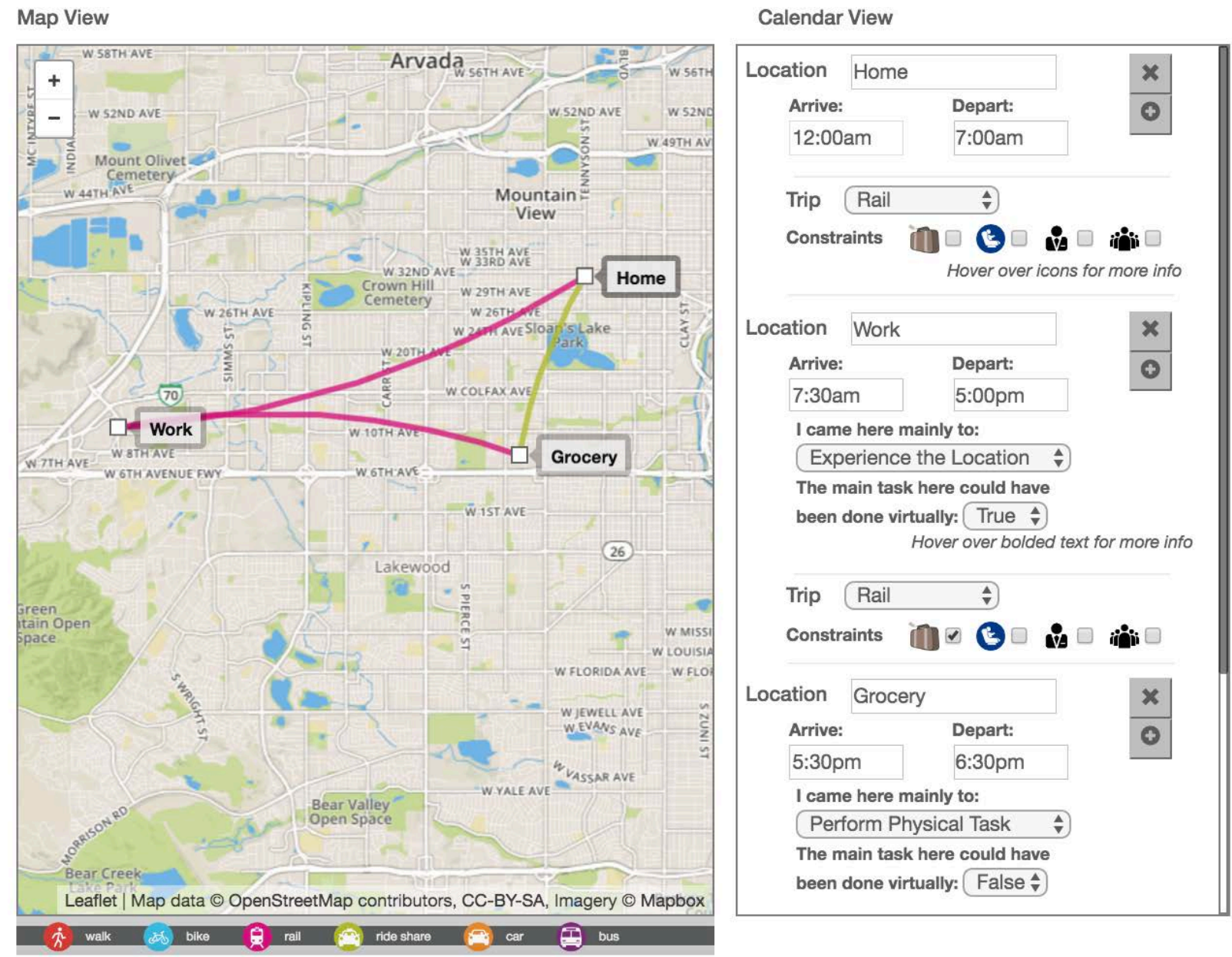

Figure 3. Travel diary interface with map and calendar

For each trip check boxes next to icons with descriptive text on mouse hovering also captured travel constraints. These limitations included the need to carry baggage, have access to a baby car seat, transport passengers or arrive at a destination in professional attire. Arrival and departure times were entered in specially formatted time drop-down boxes that defaulted to halfhour increments. Users did, however, have the ability to manually enter times at more refined intervals. Future iterations of this tool would enforce that users include a trip home at the end of the day, or validate that they started and ended the day at different locations. 


\section{User Initial Survey: Travel Attitudes \& Behaviors and Feedback}

After completing the Travel Diary, users provided feedback on the training process and data collection tools. Each user then responded to information necessary to build a basic mobility profile (car/bike ownership, cost of local bus and train rides, etc.) and calculate calorie burn while walking and biking (height, weight, age, gender). A survey measured on a seven-point Likert scale also collected information on user mode preferences, frequency of mode use and attitudes toward sustainable transportation. The survey was based on questions proposed by Jariyasunant et al. (2015). Refer to Section 7.2.2 for the full list of survey questions.

\section{User Travel Recommendation Dashboard Review}

One or more days after entering data, users were provided a link to an online dashboard displaying the travel diary information as well as five sets of daily travel recommendations, each optimized for time, cost, calories, carbon emissions or user preference. For details on how these were generated, see the Section 3.2. Upon arriving at the website, users had access to a table summarizing the outcomes for each package. Figure 4 provides an example of this interface.

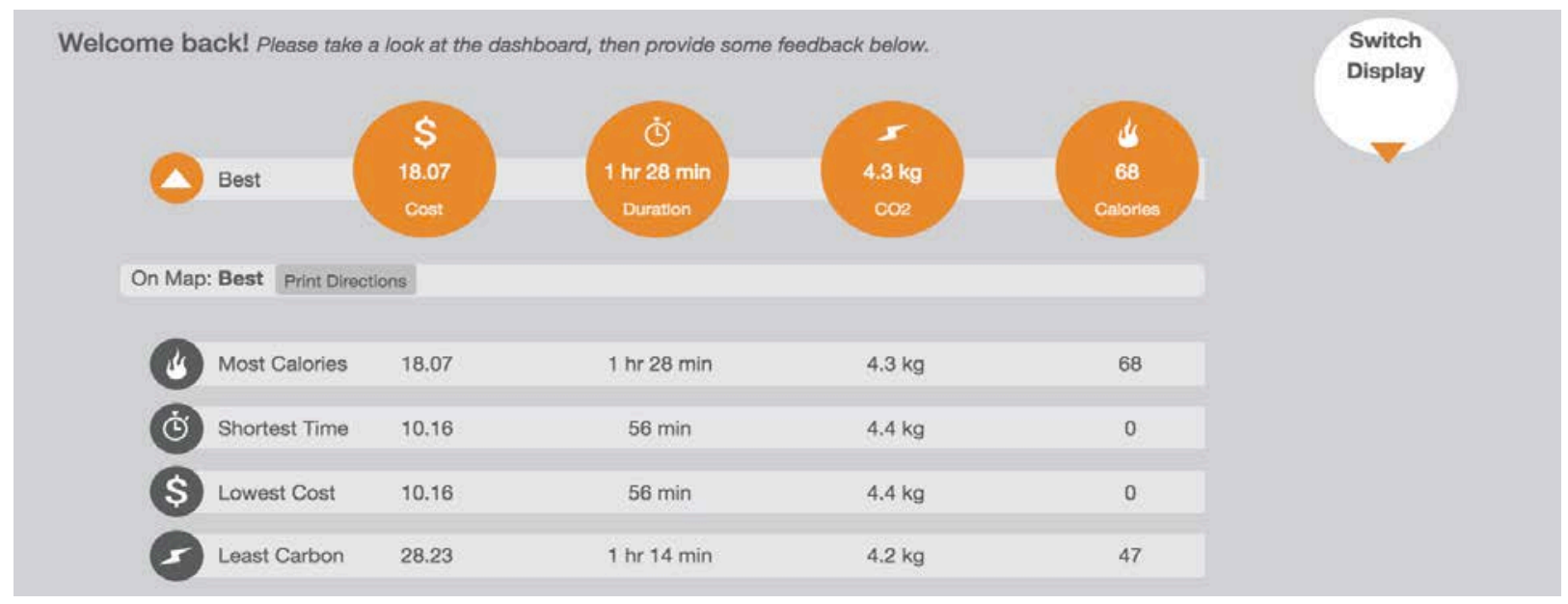

Figure 4. Personal travel dashboard showing a summary of all itineraries

Users could click on a package to see its details. Figure 5 is an illustrative example showing a map on the left and a timeline on the right. These two views were linked such that hovering over a route on the map would highlight the same route in the timeline and vice versa. Users could switch back and forth between packages using the package names to the left of the map. Clicking on routes in the map provided information about trip cost, duration, carbon emissions, and caloric intensity as well as stop and line details for public transit modes. Printable step-by-step directions were also provided for each itinerary. After viewing results, users provided feedback about their experience and responded to the same travel attitude and behavior questions as the initial survey (See Section 7.3). 


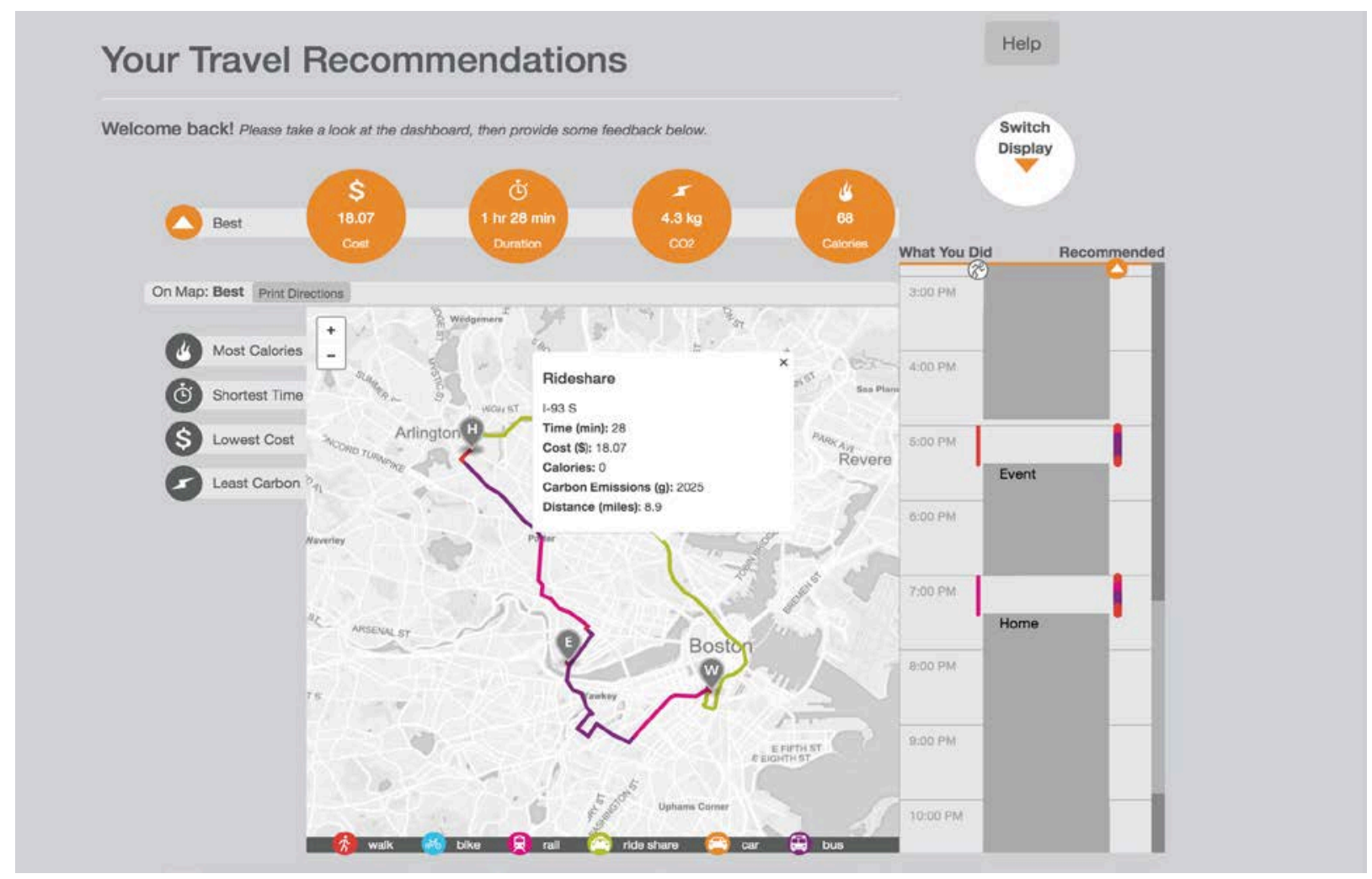

Figure 5. Personal travel dashboard showing an itinerary optimized for user preference

\section{Final Survey}

A week after viewing the dashboard, users were again sent a link to a final survey about travel attitudes and behaviors (See Section 7.4). This survey was to measure the persistence of attitude and behavior changes.

\section{Data Processing Procedures}

Programmable tasks facilitated user progression from the initial survey to the personalized dashboard and to the final survey. After the travel diary was complete for each user, automated procedures requested routing data from location-based APIs and triggered the itinerary optimization algorithm to run when all API data had been received. Metrics tracking changes to the state of user records (completion of trip itinerary optimization, completion of survey, etc.) also scheduled notifications to be sent inviting users to return to the site.

\subsection{Task Scheduling}

A Python script continuously monitored the MongoDB database looking for updates to user data. When trips in travel diaries for which no location information exists were found, the Advanced Python Scheduler instantiated a job to run on the next weekday at the same time of day as the trip's departure. The function of this job was to call the Google Directions API for walking, biking, driving, and public transit directions, as well as to the Uber API for rideshare fee and wait time estimates. Once all user trips in a travel diary were found to have complete travel information, the recommendations were assembled using the algorithm described in Section 3.2. 
The queuing script also sent payment and notifications to users using functionality provided by the Python boto package. Furthermore, the script scheduled web links to the personalized dashboard and final survey to be sent to workers. Scheduling ensured emails were sent within 5 minutes of noon.

\subsection{Trip Generation Algorithm}

Once all necessary trip information has been procured, the daily trip recommendation algorithm structured user preferences and constraints, trip origin-destination pairs, and daily event information within a hierarchy of programming objects. Data from multiple external sources can be used together once translated into this generalized framework.

\subsubsection{Algorithm Vocabulary}

The algorithm organizes the varied data associated with travel itinerary generation into the following constructs. Before discussing the details of the algorithm, a few terms need to be defined.

AlgData

An AlgData object is a container for all user information. It stores one AlgUser object, as well as Day objects that can be referenced according to their date.

\section{AlgUser}

An AlgUser object contains information about unique user attributes including height, weight, age, gender, bike/car ownership, bus/train fare and weighting coefficients for quantifying the favorability of mode alternatives.

Day

A Day object is a container for all user trips and tours on a single day. It also contains logic for performing functions that require multiple trips or tours as inputs. Such functions include calculating optimal travel itineraries and exporting this data to a format compatible with the web dashboard. 


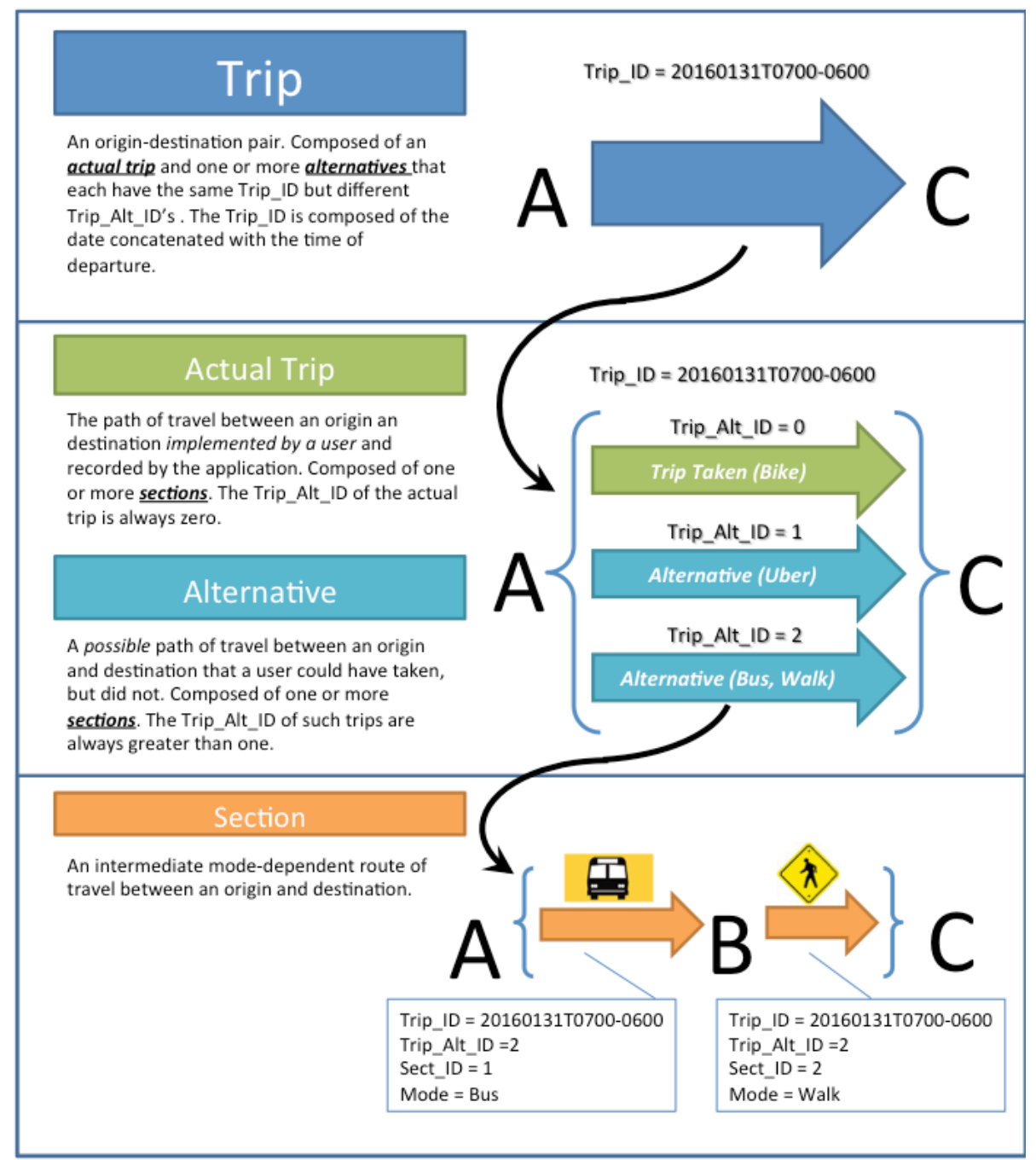

Figure 6. Relationships between trips, trip alternatives, and sections

Trip

A Trip object is at the top of a travel information hierarchy that contains Trip Alternatives (mode options for connecting locations) and Sections (Routing information for a Trip Alternative) (Figure 6). A Trip is defined by an origin destination pair and referenced according to an identifier in the format $<$ MMDDYYYY $>$ T $<$ HHMMSS $>$ - $<$ HHMM for UTC offset $>$.

\section{Trip Alternative}

A Trip Alternative object stores one or more Section objects referenced by sequentially increasing identifiers. A Trip Alternative represents one feasible alternative for travelling from an origin to a final destination and contains logic for aggregating route attributes from multiple Sections. A trip actually implemented by the AlgUser is required and distinguished by the lowest trip alternative identifier value. 


\section{Section}

A Section contains information and functionality for computing all metrics pertaining to one stage of a Trip Alternative. Attributes include time, cost, caloric intensity, carbon emissions, user preference score, start time, end time, and mapping coordinates.

\section{Loop}

A Loop object stores a series of Trips that begin and end at the same destination. Nested Loops are related to one another through parent-child relationships. For example, the series of destinations depicted in Figure 7 shows the sequence Home, Work, Lunch, Work, Home which consists of one parent (Home to Work to Home) and one child (Work to Lunch to Work). The code is capable of handling arbitrarily deep hierarchies of parent-child loops, though rarely did we encounter daily travel patterns more than one level deep. However, more complex relationships could arise if the timeframe under consideration were extended beyond a single day.

\section{Parent-Child Loop Relationships}

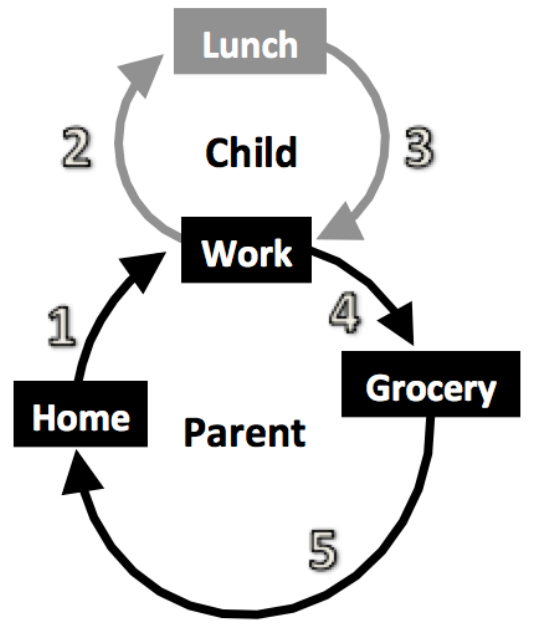

Figure 7. Relationships between parent and child loops

\section{Mode Set}

A Mode Set is a constraint on the modes available to complete trips within a loop. This paper examines three Mode Sets; being 'car', 'bike' and 'unencumbered'. 'Car' Mode Set limitations restrict personal travel to transport by personal vehicle. Once a Loop is started using a car, a car must be used for all proceeding trips within that Loop (but not necessarily for trips in child loops). Logically this constraint serves to restrict the car from being abandoned at an intermediate location along the Loop and to ensure that once a Loop is started without a car, the car will not be available until the traveler returns to a location at which the vehicle would plausibly reside. The 'bike' Mode Set logic enforces similar constraints except for bicycle commuters. Public Transportation modes are also compliant with the 'bike' Mode Set, with the understanding that bicycles can be transported on most major bus and train lines. Finally, the 'unencumbered' Mode Set permits walking, public transportation (including bus, train and ferry) and rideshare options. 


\section{Calendar Event}

A Calendar Event is an intermediate location (or the final location) along a Loop with a fixed arrival and departure time. Calendar Events can also be associated with one or more limitations for the preceding trip, including the need to transport baggage, a baby (in a car seat) or passengers, or to arrive in professional attire (and presumably not sweaty from exertion while travelling). Arrival at Calendar Events is granted a lateness threshold, defaulted to 10 minutes, to account for some flexibility in scheduling.

\section{Scenario}

A Scenario is a unique combination of a Loop with a Mode Set. It represents one possible sequence of mode restriction for all Trips in a Day. A Scenario is feasible for a user to implement (based on car/bike ownership and logical constraints inherent to Loops) and results from recursive branching at all parent-child Loop junctions as outlined in Section 3.2.3 and illustrated in Figure 7.

\subsubsection{Algorithm Data Management}

Figure 8 illustrates the hierarchal relationship between the terms outlined in the previous section. For trip data, Day, Trip, Trip Alternative and Section objects are stored within their respective containment objects (Sections are stored in Trip Alternatives, Trip Alternatives in Trips, etc.) according to their unique identifiers. Likewise, calendar event data is stored within Day objects. In this way, methods called on Days can access all necessary data for optimization calculations through cascading references to lower level objects. 


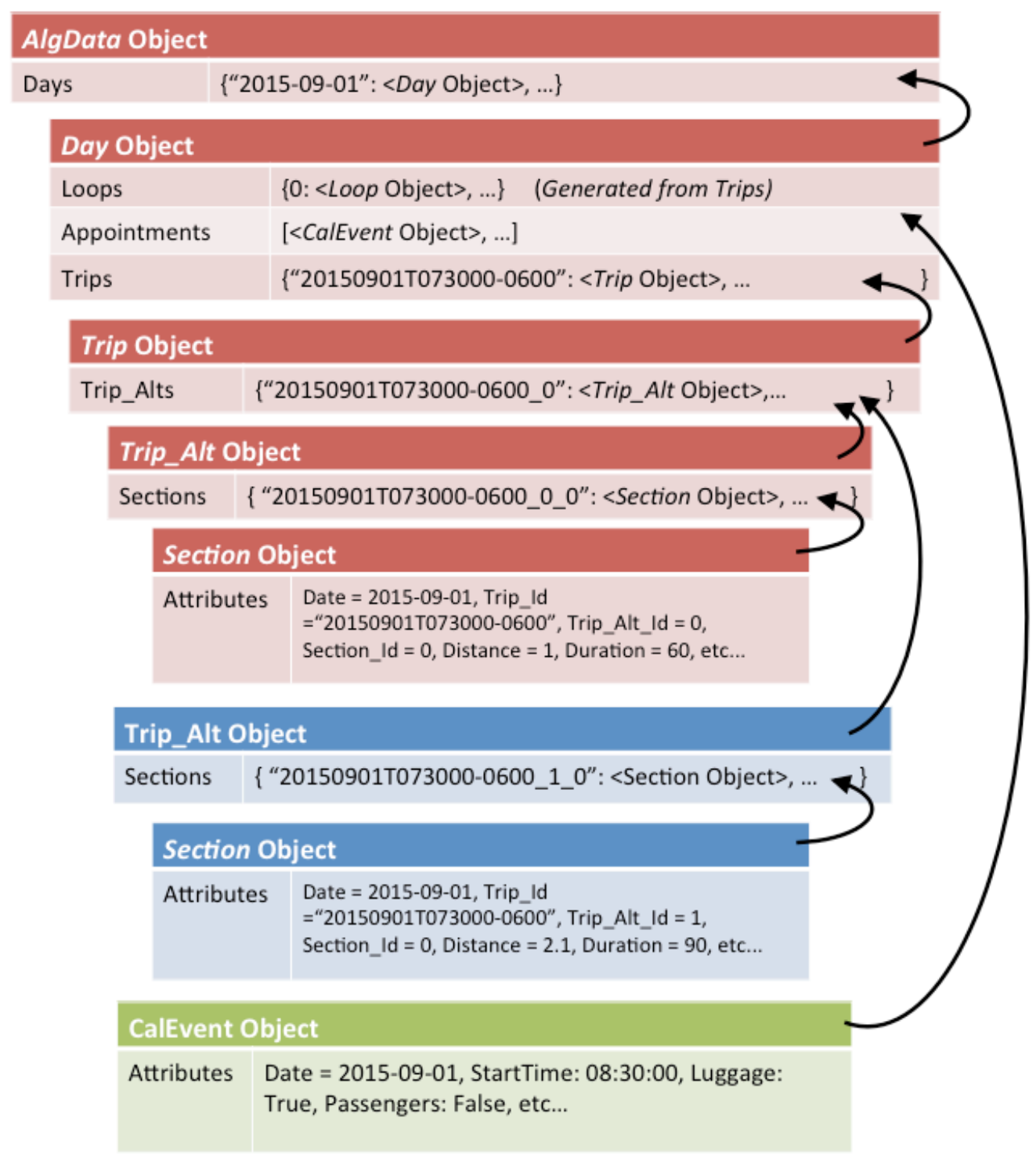

Figure 8. Data management hierarchy

\subsubsection{Recommendation Generation}

The algorithm begins by organizing the data collected from scheduled tasks (See Section 3.1) into Days, Trips, Trip Alternatives and Sections. It then analyzes the travel diary and identifies Loops. All Trip Alternatives are then filtered according to the logical constraints outlined in Table 1. 
Table 1. Logic for Filtering Trip Alternatives

\section{A Trips Alternatives is excluded if...}

Start or end time overlaps with another trip actually made by a user on the same day or a calendar event*

The distance is longer than the user's travel threshold for the mode (walking and cycling only)

The user does not own a necessary mobility asset (car and cycling only)

Requires biking or long walks before a professional meeting

Requires anything but car after a car seat is required **

Requires anything but car or ride share after a passenger pickup **

Requires anything but car, public transit or ride share after luggage pickup **

* If the alternative is not public transit, the trip will be set back in time if doing so will not cause Trip or Calendar Event conflicts rather than be deleted

** Constraints are lifted once the user completes the loop on which the calendar event occurs

Next, considering the 'car', 'bike' and 'unencumbered' Mode Sets independently, the algorithm begins by filtering Trip Alternatives on the parent Loop based on the Mode Set's particular mode restrictions. When child Loops are encountered, the algorithm must branch to consider the scenario where the child Loop is completed under the same mode set as the parent, as well as any other scenarios for viable alternate mode sets. For example, if a traveler commences a parent Loop using a bike or car, he or she has the opportunity to switch to unencumbered modes at the start of a child Loop, because the bike or car can be parked at this intermediate location. If the parent Loop is commenced without a bike or car however, child tours have no viable alternate. Branching at all parent-child Loop junctions yields a full set of viable daily travel scenarios as depicted in Figure 9.

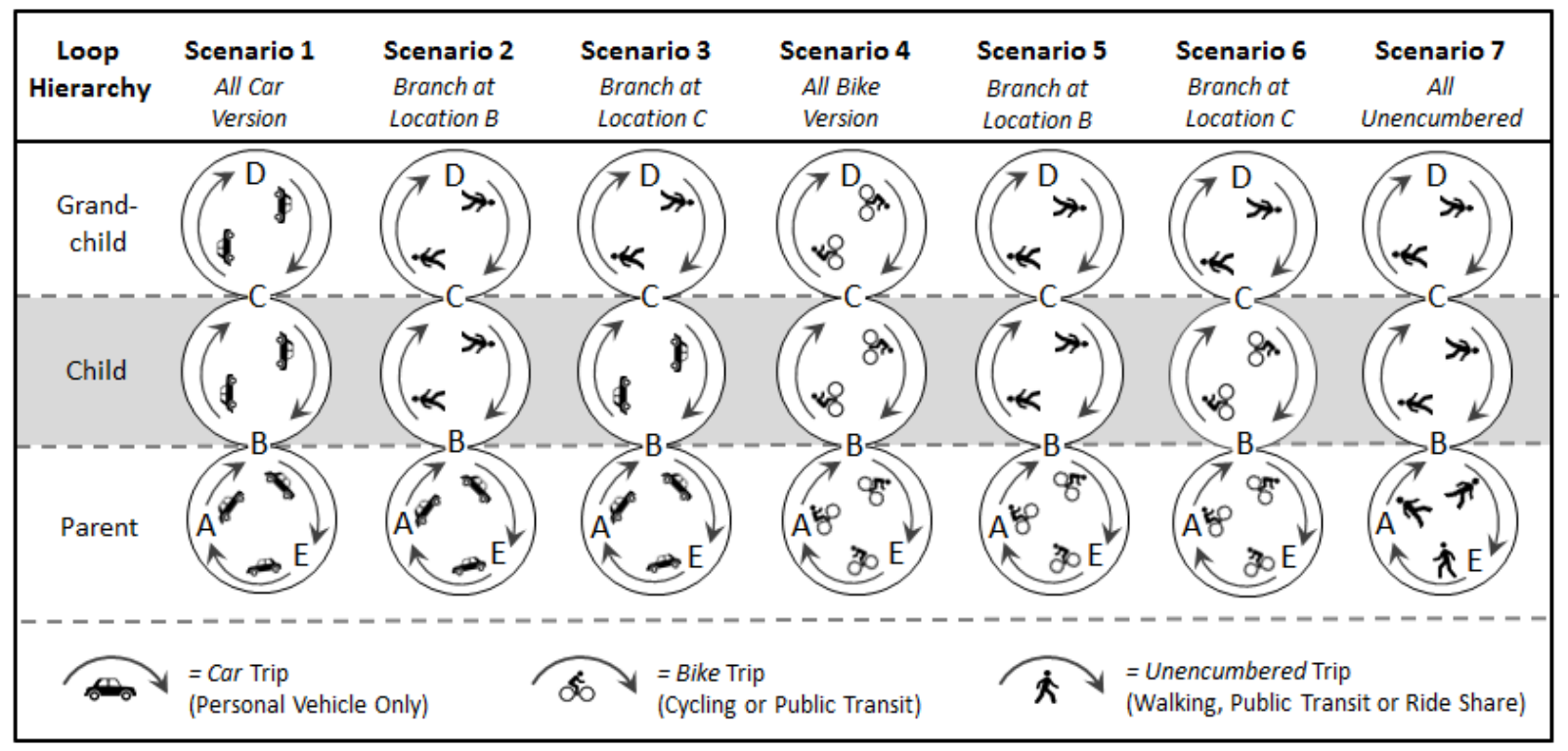

Figure 9. Possible travel scenarios based for one pair of parent-child loops 
After all possible travel scenarios have been identified, each scenario is assessed for total estimated cost, duration, carbon dioxide emissions, caloric expenditure and user preference score. Information about the assumptions in calculating these metrics is described in Section 3.3. Cumulative metrics for each scenario are aggregated by independently considering each Trip (for which Trip Alternatives have been filtered by a Mode Set's mode restrictions) and adding the optimal metric from the optimal Trip Alternative. When optimizing for time, cost, or carbon dioxide, the Trip Alternative that yields a minimum value is considered optimal. For caloric intensity and user preference, maximum values are considered optimal.

Finally, for each metric, the scenario that yields the optimal cumulative metric is identified and converted into format for visualization as a set travel recommendation on the user's personalized dashboard.

\subsection{Trip Metric Calculations}

This section catalogs the assumptions made in calculating the time, cost, caloric intensity, carbon emissions and user preference for each trip.

\subsubsection{Time}

For trips using walking, cycling, driving or public transit travel mode, the Google Directions API provided trip duration estimates in seconds. For more information about the Google Directions API, refer to https://developers.google.com/maps/documentation/directions/start .Trip ride-share duration was calculated as the sum of the duration for a car trip between the origin and destination in seconds, as provided by the Google Directions API, and the estimated wait time in seconds for a driver to arrive at the destination, as provided by the Uber API. For more information about the Uber API, refer to https://developer.uber.com/docs/rush.

When conveyed to the user, durations were rounded to the nearest hour and minute.

\subsubsection{Cost}

Walking and biking alternatives were assumed to be free.

Car trip costs were calculated by dividing the total distance in miles by a standard gas mileage rate of 38.92 passenger-miles per gallon gasoline (Oak Ridge National Laboratory 2014). In choosing passenger-mile units of measurement the authors intend to account for the fact that drivers on average will at least occasionally ride with passengers. Assuming that costs are shared among drivers and passengers renders an automotive cost estimate that is most competitive with alternative travel modes. The resulting required amount of gasoline in gallons derived from the initial calculation was then multiplied by a national average for the price of regular gasoline on the day of the calculation, as obtained from a web-service hosted by the Department of Energy and U.S. Environmental Protection Agency at http://www.fueleconomy.gov/ws/rest/fuelprices.

Ride-share costs were derived from the average of the high and low fare estimates provided by the Uber API.

In the initial survey (Section 7.2 - Travel Preferences and Constraints), users of the platform were asked to provide the cost of the next bus or train ride. Commute pass holders were instructed to enter a value of $\$ 0$ to signify a free trip. User-provided values, when available, were used to estimate trips making use of public transit. When users did not provide this information, 
price information from the Google Directions API provided fare estimates. Since the Google Directions API does not provide fare estimates for all metropolitan areas, in cases where public transit fares were unknown, bus rides were assumed to cost $\$ 2.25$ and train rides $\$ 4.00$. All ferry rides were assumed to cost $\$ 10.00$.

\subsubsection{Carbon Emissions}

Walking and biking alternatives were assumed to have zero carbon emissions.

Car tailpipe carbon emissions were calculated by dividing the total distance in miles by a standard gas mileage rate of 38.92 passenger-miles per gallon gasoline (Oak Ridge National Laboratory 2014). Again, basing the calculation in passenger-miles assumes emissions are distributed between drivers and passengers. The intention is to render a more competitive carbon emission estimate than assuming the participant is always accountable for all emissions while travelling in a car. The resulting required amount of gasoline in gallons was multiplied by 8887 grams of carbon dioxide per gallon of regular gasoline (U.S. Environmental Protection Agency 2016).

Ride share emissions were calculated the same as private vehicle emissions, except that the distance was increased by the product of the wait time, in hours, and an assumed average approach speed of 10 miles per hour.

When public transit vehicle information is available, transit emissions are calculated by dividing the distance in miles by the passenger-miles per gallon gasoline equivalent of the vehicle (See Table 2) (Oak Ridge National Laboratory 2014). When vehicle information is unavailable the average fuel economy of all vehicle types was used.

Table 2. Emission Calculation Assumptions

\begin{tabular}{ll}
\hline Vehicle Type & Mileage (p-mpg) \\
\hline Bus & 30.83 \\
Metro Rail, Subway, Tram, Monorail & 51.82 \\
Rail, Heavy Rail, Commuter Train & 43.79 \\
\hline
\end{tabular}

\subsubsection{Calories}

Calorie expenditure calculations for the walking and biking modes were derived from estimates of basal metabolic rates (Harris and Benedict 1918) and summarized in Table 3.

Table 3. BMR Calculations

\begin{tabular}{lc}
\hline Gender & BMR Calculation \\
\hline Male & $13.75 \times$ Weight $(\mathbf{k g})+5 \times$ Height $(\mathbf{c m})-6.76 \times$ Age +66 \\
Female & $9.56 \times$ Weight $(\mathbf{k g})+1.85 \times$ Height $(\mathbf{c m})-4.68 \times$ Age +655 \\
\hline
\end{tabular}


User BMR was multiplied by a metabolic equivalent (MET) of 3.3 for walking (Royal et al, 2008) and 4 for biking (Ainsworth et al, 2000). In cases of insufficient data for these calculations, user records were excluded from reported results.

In future iterations of the platform would implement average calorie burns of 76 calories per mile walking and be 27 calories per mile cycling. These average values reflect resting metabolic rates of 1613 and 1563 calories per day for men and women respectively (Arciero et al. 1993), MET values of 3.3 and 4 for walking and cycling, and corresponding rates of speed of $3 \mathrm{mph}$ and $9.9 \mathrm{mph}$ for walking and cycling respectively.

Car, rideshare and public transit modes were assumed to have no caloric expenditures, though future iterations should calculate a resting metabolic for the duration of the trip. The zero calorie assumption is not expected to significantly effect optimization calculations, since one would be expected to always have higher MET rates on average while walking or cycling, than while at rest.

\subsubsection{User Preference Score}

Calculation of a trip's user preference score was based on eigenvector-derived weighting inputs to the Analytic Hierarchy Process (AHP) (Ishuzaka and Nemery 2013). Users were asked to indicate preference between all pairwise combinations of trip optimization metrics, being time, cost, calories and carbon emissions. Values derived from these preferences were translated into quantitative values according to the scheme outlined in Table 4.

Table 4. AHP Pairwise Metric Weighting

\begin{tabular}{ll}
\hline Preference Range & Value \\
\hline Extremely Prefer Metric 1 & $1 / 5$ \\
\hline Highly Prefer Metric 1 & $1 / 4$ \\
\hline Prefer Metric 1 & $1 / 3$ \\
\hline Somewhat Prefer Metric 1 & $1 / 2$ \\
\hline Indifferent & 1 \\
\hline Somewhat Prefer Metric 2 & 2 \\
\hline Prefer Metric 2 & 3 \\
\hline Highly Prefer Metric 2 & 4 \\
\hline Extremely Prefer Metric 2 & 5 \\
\hline
\end{tabular}

Eigenvectors from a matrix provided metric coefficients for the following equation:

$$
\begin{aligned}
& \mathrm{P}=\mathbf{C}_{\mathrm{T}} \times \text { Duration (s) }+\mathbf{C}_{\mathrm{C}} \times \mathbf{C o s t}(\$)+\mathbf{C}_{\mathrm{GHG}} \times \text { Carbon Emissions (g) }+\mathbf{C}_{\mathrm{CAL}} \times \text { Calories (cal) } \\
& \text { Where: } \\
& \mathrm{P}=\text { User Preference Score } \\
& \mathrm{C}_{\mathrm{T}}=\text { Time Coefficient } \\
& \mathrm{C}_{\mathrm{C}}=\text { Cost Coefficient } \\
& \mathrm{C}_{\mathrm{GHG}}=\text { Carbon Emission Reduction Coefficient } \\
& \mathrm{C}_{\mathrm{CAL}}=\text { Calories Coefficient }
\end{aligned}
$$




\section{User Experience Feedback}

265 AMT workers provided completed responses to the first stage of the Trip Itinerary

Optimization platform. About $74 \%$ of these platform users returned to view the personal mobility dashboard and 65\% returned after that to complete the Final Survey. As users progressed through the application as described in Section 2.1.3, feedback about their experience was collected. The responses to each component of the application will now be described.

\subsection{Instructions}

When asked, "How good were the instructions?" the average user response was between "Somewhat Good" and "Very Good" (3.4 out of 4). Numerous users commented that the 12-slide format was tedious. Users also commonly suggested that the training could be improved by structuring it as a video rather than a series of slides or by providing a 'sandbox' environment for practicing data entry. Future iterations of the interface should also make instructions more clear about how to add the final return trip home for each day, and contain functionality to validate this condition.

\subsection{Data Entry}

When asked, "How easy were the previous two Data Entry pages to use?" users typically rated the data entry pages between "Somewhat Good" and "Very Good" (3.4 out of 4). In open-ended responses soliciting additional feedback, platform users commonly noted that they found the data entry task enjoyable and interesting.

\subsection{Personal Dashboard}

Those who used the platform generally agreed on a seven-point Likert scale that using the dashboard was easy ( 4.8 on scale from 0 of $6 ; 3$ being neutral) and enjoyable (4.7), and also that it would be helpful in adopting new transportation behaviors (4.5). In free responses to requests for additional feedback, users expressed a desire for high-resolution and real-time traffic and weather forecasts, identification of food stops along routes, and the visualization of pedestrian amenities (sidewalks, trees and shortcuts through alleys). Reponses also suggested a more comprehensive explanation of the derivation of optimal metric calculations would improve the experience. Several users noted that the cost for car travel seemed high, indicating that the true cost of car ownership may not be factored in to decision they make about car travel. Future versions should include a brief explanation of the full cost of car ownership for those that care to know more. For instance, an information icon could be placed next to the cost column in Figure 3 that provides a pop-up with cost calculation details.

\subsection{Final Survey}

When asked for additional comments, numerous users indicated that they lack access to reliable public transportation options and would like to see more alternatives shown in the dashboard (or recommendations on what do if alternatives are not available). Other comments followed similar trends discussed regarding the Personal Dashboard. 


\section{Trip Itinerary Optimization Effectiveness}

Data collected from users of the Trip Itinerary Optimization platform provides travel pattern metrics that provide insight into typical travel attitudes and behaviors, as well as the effectiveness of a tool like this in motivating behavior change.

\subsection{Simplification of Travel Possibilities}

Platform users recorded an average of 2.9 trips per day and 31 of the 265 travel diaries collected contained only one trip. Furthermore, 58 users did not record a single repeated destination, suggesting that a final return trip home was not entered and the number of trips per day that users actual travelled is higher. Among platform users who did have one or more repeated destination, the average number of trips was 3.2 per day. About one trip per respondent contained a baggage, passenger or professional appearance constraint.

The 197 Platform users who returned to view the personal dashboard on average viewed 1.6 unique trip itineraries reduced from 66 possible mode to trip combinations. $45 \%$ of dashboard viewers saw the one itinerary, typically driving to all destinations, repeated five times as the recommendation for each optimization metric because no feasible alternatives were available.

\subsection{Response to Recommended Itineraries}

Users found that the time, cost and preferred packages were most intuitive, and they expressed the greatest willingness to actually implement these itineraries within their daily travel routines (Table 5). Alternatively, users found the calories and carbon-optimized itineraries intuitively, but did not demonstrate strong willingness to try either recommendation.

Table 5. Reponses to Recommended Itineraries while Viewing Dashboard

\begin{tabular}{lcc}
\hline $\begin{array}{l}\text { Recommendation } \\
\text { Set }\end{array}$ & $\begin{array}{l}\text { "This set of trip } \\
\text { recommendations makes } \\
\text { sense to me." }\end{array}$ & $\begin{array}{l}\text { "I will try this } \\
\text { recommendation." }\end{array}$ \\
\hline Time & 5.0 & 4.7 \\
Cost & 4.8 & 4.4 \\
Calories & 4.0 & 3.4 \\
Carbon & 4.2 & 3.7 \\
Preferred & 4.7 & 4.5
\end{tabular}

$13 \%$ of the 171 users who returned to complete Final Survey reported changing travel behaviors in response to seeing the dashboard. Nine users tried the time-optimized itinerary and the calories- and carbon-optimized itineraries were each implemented by eight respondents. Also, five tried the cost optimized itinerary and 3 tried the user preference optimized alternative. 


\section{Conclusion}

The application described in this paper provides a preliminary framework for an automated personal mobility assistant. Through reducing barriers to data collection and automating optimization analysis, such tools can better prepare individuals to achieve optimal travel outcomes. Those exposed to the trip recommendation platform generally enjoyed the experience of using it and recognized its value. Even at this early stage the application demonstrated its ability to facilitate automated trip planning, and $13 \%$ of users reporting changing their travel behavior in response to it.

Results indicate also that users retained a strong preference for optimizing time and cost, over caloric intensity and carbon emissions. Future work will examine additional methods for quantifying the co-benefits of sustainability-oriented itineraries. Additional information (such as elevation) and the enhancement of routing algorithms for calorie burn optimization may also help improve the benefit they provide to travelers.

The problem of selecting a user's optimal itinerary is dynamic and complex. This preliminary study incorporated historic traffic condition data, public transit timetables, bike path routes, and other pertinent travel information available via web API's. Open-ended responses suggest, however, that enhanced metrics and visualizations on the relative safety and pedestrian amenities (sidewalks, shade, shopping opportunities) of walking and biking routes would also help to reduce perceived barriers to non-car oriented modes. Users also commonly expressed a desire to model have the ability to interactively model adjustments to travel times and to be made aware of more varied (even if suboptimal) itineraries. In general, more sophisticated information and functionality will improve recommendation accuracy and promote more widespread adoption.

The Trip Itinerary Optimization (TrIO) platform provides mechanisms for including additional transportation modes including motorcycles, short-term car rentals, bike and scooter shares, and other shared mobility services. Inclusion of such alternatives will be increasingly important in making travelers aware of the relative tradeoffs between modes they may have not used before, and in ultimately helping them to achieve their personal goals. The application proposed in this paper moreover provides a first step towards the development of more powerful capabilities that enable transportation managers to more personally engage with travelers in order to improve system performance in aggregate. Future development of TrIO will seek to take enhance personalization and connectivity with richer datasets to provide information that leads to quality of life improvements, better use of existing infrastructure, and reduced environmental impacts. 


\section{Glossary}

Section

Trip Alternative

Loop

Loop (Parent)

Loop (Child)

Mode Set

Scenario
A continuous portion of a Trip making use a single mode. A Trip that involves walking, taking the bus and then walking again to the destination consists of three distinct sections.

A possible combination of mode-specific portions, or Sections, to connect an origin to a final destination.

A series of Trips that begin and end at the same location.

A parent Loop contains one or more destinations, aside from the initial Trip origin, a traveler repeatedly visited. In the series of locations Home, Work, Lunch, Work, Home, the location 'Work' is repeated such that the sequence Home, Work, Home is a parent Loop.

A child Loop emanates from the repeated location in a parent Loop. In the series of locations Home, Work, Lunch, Work, Home, the location 'Work' is repeated such that the sequence Work, Lunch, Work is a child Loop.

A set of mode restrictions for completing all Trips within a Loop.

All possible combinations of Loops and Mode Sets. 


\section{References}

Ainsworth, Barbara E., William L. Haskell, Melicia C. Whitt, Melinda L. Irwin, Ann M. Swartz,

Scott J. Strath, William L. O'Brien, et al. 2000. "Compendium of Physical Activities: An Update of Activity Codes and MET Intensities." Medicine \& Science in Sports \& Exercise 32 (9 Suppl.): 498-504. doi:10.1097/00005768-200009001-00009.

Amazon.com. 2013. AWS SDK for Python, Boto (version 2.7).

https://pypi.python.org/pypi/boto/2.7.0.

Anagnostopoulou, Evangelia, Efthimios Bothos, Babis Magoutas, Johann Schrammel, and Gregoris Mentzas. 2016. "Persuasive Technologies for Sustainable Urban Mobility." arXiv Preprint arXiv:1604.05957.

Arciero, Paul J., Michael I. Goran, and Eric T. Poehlman. 1993. "Resting Metabolic Rate Is Lower in Women than in Men." Journal of Applied Physiology 75 (6).

Berinsky, Adam, Gregory Huber, and Gabriel Lenz. 2012. "Evaluating Online Labor Markets for Experimental Research: Amazon.com's Mechanical Turk.” Political Analysis 20 (3): 351-68.

Binsted, Anne, Owen Waygood, Anna Clark, and Erel Avineri. 2011. "Communicating the Impacts of Transport Choices to Encourage Low Carbon Travel Behaviours." In Proceedings of the 8th SoNorA (South-North Axis) University Think Tank Conference, 1868:5-17. Szczecin, Poland. http://www.sonoraproject.eu/Febe/university/file/120/Proceedings.pdf.

Fujii, Satoshi, and Ayako Taniguchi. 2006. "Determinants of the Effectiveness of Travel Feedback Programs - a Review of Communicative Mobility Management Measures for Changing Travel Behaviour in Japan.” Transport Policy, 339-48.

Harris, J. Arthur, and Francis G. Benedict. 1918. "A Biometric Study of Human Basal Metabolism." Proceedings of the National Academy of Sciences 4 (12): 370-73.

Huff, Connor, and Dustin Tingley. 2015. “'Who Are These People?' Evaluating the Demographic Characteristics and Political Preferences of MTurk Survey Respondents." Research and Politics 1 (12). doi:10.1177/2053168015604648.

Ishizaka, Alessio, and Philippe Nemery. 2013. Multi-Criteria Decision Analysis, Full Aggregation Approach. Chichester, UK: John Wiley \& Sons Ltd.

Jariyasunant, Jerald, Maya Abou-Zeid, Andre Carrel, Venkatesan Ekambaram, David Gaker, Raja Sengupta, and Joan L. Walker. 2015. "Quantified Traveler: Travel Feedback Meets the Cloud to Change Behavior.” Journal of Intelligent Transportation Systems 19 (2): 109-24. 
Meloni, I., B. Sanjust, G. Delogu, and E. Sottile. 2014. "Development of a Technological Platform for Implementing VTBC Programs.” Transportation Research Procedia 3.

Oak Ridge National Laboratory. 2014. Transportation Energy Data Book Table 2.12. 33rd ed.

Royall, Penelope Slade, Richard P Troiano, Melissa A. Johnson, Harold W. Kohl, and Janet E. Fulton. 2008. "Appendix 1. Translating Scientific Evidence About Total Amount and Intensity of Physical Activity Into Guidelines.” In 2008 Physical Activity Guidelines for Americans, 5457.

Poushter, Jacob. 2015. "Car, bike or motorcycle? Depends on where you live." Pew Research Center http://www.pewresearch.org/fact-tank/2015/04/16/car-bike-or-motorcycle-depends-onwhere-you-live/.

psiTurk. 2014. An open platform for science on Amazon Mechanical Turk. https://psiturk.org.

Twisted Matrix Labs. 2014. Twisted (version 13.2.0).

http://twistedmatrix.com/trac/wiki/TwistedProject .

U.S. Census Bureau. 2015. “2015 American Community Survey 1-Year Estimates.” http://www.census.gov/programs-surveys/acs/technical-documentation/table-and-geographychanges/2015/1-year.html.

U.S. Department of Transportation. 2015. "Passenger Travel Facts and Figures 2015". https://www.rita.dot.gov/bts/sites/rita.dot.gov.bts/files/PTFF_Complete.pdf.

U.S. Environmental Protection Agency. 2016. "GHG Equivalencies Calculator - Calculations and References." https://www.epa.gov/energy/ghg-equivalencies-calculator-calculations-andreferences. 


\section{Survey Questions}

This section contains a list of questions and response options at each stage of user interaction with the Trip Itinerary Optimization Platform.

\subsection{Check Questions}

What level of accuracy should you use in entering locations?

- Within Inches

- Within Feet

- Within a few blocks (correct)

If your main reason for going to work is to make a verbal presentation to a group of people, you should indicate your main reason for making the trip is to:

- Perform a Task

- Communicate with Others (correct)

- Experience the location

Shopping for clothes can be performed virtually because it is possible to make such purchases online:

- True (Correct)

- False

\subsection{Initial Survey Questions}

\subsubsection{Instruction Quality}

How good were the instructions?

- Very Bad (0)

- Somewhat Bad (1)

- Neither Bad or Good (2)

- Somewhat Good (3)

- Very Good (4)

What parts (if any) of the instructions were not clear, and how could they be improved?

- (Open Ended)

\subsubsection{Travel Attitudes and Behaviors}

Please rank your agreement with the following statements:

"I know how much CO2 I emit from transportation."

"I know how many calories I burn while commuting/traveling."

"I know how much money I spend on commuting/traveling per year."

"I know how much time I spend commuting/traveling per year."

"I can get exercise when traveling."

"Regardless of cost, I choose the fastest way to travel."

"There are many constraints and limitations that keep me from changing my transportation behavior." 
"I don't see why one would engage in sustainable behavior in transportation." "We should raise the price of gasoline to reduce congestion and air pollution." "I feel guilty if I don't take sustainable modes of transportation."

"Using sustainable modes of transportation is beneficial to my health."

"Engaging in sustainable transportation behavior is very important to me."

"I value the health benefits of using sustainable modes of transportation."

"Greenhouse gases cause environmental problems such as global warming."

"Everybody together should reduce the amount of fuel burned as a result of their transportation behavior."

"I like":

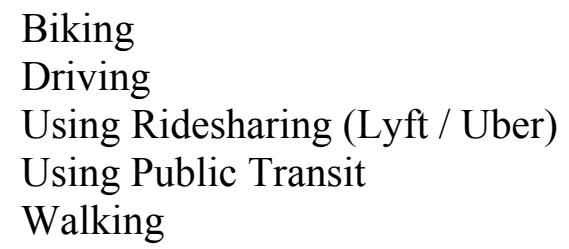

- Entirely Disagree (0)

- Mostly Disagree (1)

- Somewhat Disagree (2)

- Neither Agree nor Disagree (3)

- Somewhat Agree (4)

- Mostly Agree (5)

- Entirely Agree (6)

\section{Over the next few months, how often do you intend to:}

Bike

Drive

Use Ridesharing (Lyft / Uber)

Use Public Transit

Walk

Frequency based on a seven point Likert scale:

- Never (0)

- Rarely (1)

- Occasionally - Monthly (2)

- Sometimes - Weekly (3)

- Frequently - Few Times a Week (4)

- Almost Always (5)

- Always (6) 


\subsubsection{Travel Preferences and Constraints}

How often do you follow the travel pattern you just submitted?

- I do that every weekday (0)

- I do that some days of the week (1)

- I do that every now and then (2)

- I only did that once (3)

For what day of the week did you enter information?

- Days of Week dropdown

Do you own a bike?

- True

- False

Do you own a car?

- True

- False

What kind of fuel does your car use?

- Electric

- Gasoline

- Diesel

- Other

How much would your next bus ride cost?

- (Open-ended)

How much would your next train ride cost?

- (Open-ended)

What is your weight in pounds?

- (Open-ended)

What is your height?

- (Open-ended)

What is your age?

- (Open-ended)

What is your gender?

- (Open-ended) 
Given two travel outcomes below, please indicate which you prefer more.

- Extremely Prefer

- Highly Prefer

- Prefer

- Somewhat Prefer

- Indifferent

\section{Pairs}

Save Time, Save Money

Save Time, Burn Calories

Save Time, Reduce Carbon Emissions

Save Money, Burn Calories

Save Money, Reduce Carbon Emissions

Burn Calories, Reduce Carbon Emissions

\subsubsection{Data Entry Experience}

How easy were the previous two Data Entry pages to use?

- Very Hard (0)

- Somewhat Hard (1)

- Neither Easy or Hard (2)

- Somewhat Easy (3)

- Very Easy (4)

What parts (if any) of the past two Data Entry pages were not clear, and how could they be improved?

- (Open Ended)

Please provide any additional feedback.

- (Open Ended)

\subsection{Post Treatment Survey}

\subsubsection{Dashboard Feedback}

Please rank the sets of travel recommendations from 'Most Likely to Try' to 'Least Likely to Try':

- Time

- Cost

- Calories

- Carbon Emission

- Best

For each set of travel recommendations, please indicate your agreement with the following statements:

"This set of trip recommendations makes sense to me." 
Hint: For example, the 'Time' set of trip recommendations looks like the fastest way for me to get between all locations.

"I will try this recommendation."

Please also indicate your agreement with the following statements:

"I enjoyed looking at the dashboard and getting a summary of my results."

"If I were trying to change my travel behavior (be greener, reduce cost, travel less), the dashboard would be helpful."

"The dashboard was easy to use."

Agreement was measured on a seven point Likert scale:

- Entirely Disagree (0)

- Mostly Disagree (1)

- Somewhat Disagree (2)

- Neither Agree nor Disagree (3)

- Somewhat Agree (4)

- Mostly Agree (5)

- Entirely Agree (6)

Aside from time, cost, calories, preference and carbon emissions, what other types of trip itinerary sets would you like to see included in the dashboard?

- (Open-ended)

Was there anything that surprised you about any of the trip recommendations?

- (Open-ended)

\subsubsection{Travel Attitudes and Behaviors}

Questions repeated from Section 7.2.2.

\subsubsection{Dashboard Design Feedback}

What additional information would improve the trip recommendations?

- (Open-ended)

How could the display or functionality of the dashboard be improved?

- (Open-ended)

Please provide any additional feedback.

- (Open-ended) 


\subsection{Final Survey}

\subsubsection{Behavior Change}

Did your travel behavior change in response to seeing the dashboard?

- Yes

- No

Please describe the change you made.

- (Open-ended)

Please check the box next to any sets of trip recommendations you used.

[For checked recommendations]

How often do you plan to keep using these travel recommendations?

- Never (0)

- Rarely (1)

- Occasionally - Monthly (2)

- Sometimes - Weekly (3)

- Frequently - Few Times a Week (4)

- Almost Always (5)

- Always (6)

What motivated you to try this package?

- (Open-ended)

What could have made the trip recommendations more helpful?

- (Open-ended)

What is your primary mode of transportation?

- Walk

- Personal Bike

- Public Transit (Bus or Train)

- Personal Car

- Rideshare (Lyft/Uber)

- Bikeshare

- Car Rental (including ZipCar, Car2Go)

- Another Person's Car

- Private Bus or Shuttle

- Other

If 'Other'

Please specify

- (Open-ended)

What mode would you use if your primary mode was unavailable?

- Same as primary mode options 
Please provide any additional feedback.

- (Open-ended)

\subsubsection{Travel Attitudes and Behaviors}

Questions repeated from Section 7.2.2. 


\section{Amazon Mechanical Turk (AMT) Platform Specifications}

Amazon Mechanical Turk (AMT) is a web-based platform through which requesters can post Human Intelligence Tasks (HIT) for workers to complete for a fixed price. HITs are commonly tasks that are simple for humans to do but hard to automate using computers, though it is increasingly used for human-focused research. Berinsky (2012) finds that AMT are more representative of the U.S. population than in-person convenience samples.

This study made use of AMT to solicit, pay and communicate with platform users. AMT worker participation was restricted to U.S. citizens. Workers provided Informed Consent in compliance with IRB standards before beginning the study, which was advertised through a HIT titled "Transportation Recommendation Study". Workers were not allowed accept the HIT more than once. Up to 15 workers were allowed to actively be working on the HIT at any one time and each worker was allowed up to one hour to submit responses before the session was expired.

Worker compensation was based on the federal minimum wage of $\$ 7.25$ and an average time for HIT completion. Accordingly, the payment was $\$ 1.75$ for completing the initial survey, $\$ 1.50$ for reviewing the information dashboard, and $\$ 1.20$ for completing the final survey. The initial payment was delivered through the AMT approval function and the remaining payments were made through AMT's bonus mechanism. From 274 initial HIT responses, the retention rate was approximately $74 \%$ for the first follow up survey and $64 \%$ for the second follow up. The AMT interface allows HITs that do not comply with stated instructions to be declined, resulting in the withholding of payment. Less than $1 \%$ of all HITs were declined during this study, and in such cases workers were notified and provided the opportunity to retake the HIT or provide additional validating information about their initial responses.

The Python boto (Amazon.com 2013) package facilitated automated interaction with the Amazon Web Services (AWS) architecture for tasks such as approving and declining HITs, as well as notifying workers of status updates. 


\section{AMT Participant Demographic Profile}

Analysis of participants involved in this study, summarized in Table 6, suggests user feedback may be biased towards a population that is younger, more urban, and has more males than the national average. Car and bike ownership rates, however, align well with national averages, particularly for those who viewed the personal mobility dashboard and completed the final survey. Those who participated in the study tended to take fewer but longer distance trips than the national average, with slightly fewer trips taken by car than would be expected. Interestingly, AMT participants living in rural areas were less likely to travel by car than those living in urban areas.

Table 6. Participant Demographics Throughout Study Phases

\begin{tabular}{|c|c|c|c|c|}
\hline & $\begin{array}{l}\text { U.S. } \\
\text { Population }\end{array}$ & $\begin{array}{l}\text { Completed } \\
\text { Travel Diary } \\
(n=265)\end{array}$ & $\begin{array}{l}\text { Viewed the } \\
\text { Personal } \\
\text { Dashboard } \\
(n=197)\end{array}$ & $\begin{array}{l}\text { Completed Final } \\
\text { Survey } \\
(n=171)\end{array}$ \\
\hline Median Age & $37.8^{1}$ & 31 & 31 & 31 \\
\hline Male & $36.5^{1}$ & 30 & 31 & 31 \\
\hline Female & $39.1^{1}$ & 31 & 32 & 33 \\
\hline Percent Female & $50.8 \%^{1}$ & $41.1 \%$ & $41.6 \%$ & $40.3 \%$ \\
\hline Percent Male & $49.2 \%^{1}$ & $58.8 \%$ & $58.3 \%$ & $59.6 \%$ \\
\hline Percent w. Car Access * & $91 \%{ }^{1}$ & $86.4 \%$ & $89.3 \%$ & $90.0 \%$ \\
\hline Percent w. Bike Access & $53 \%^{2}$ & $52.80 \%$ & $52.80 \%$ & $52.80 \%$ \\
\hline$\%$ Urban Population** & $80.70 \%{ }^{1}$ & $83.80 \%$ & $85.20 \%$ & $84.80 \%$ \\
\hline $\begin{array}{l}\text { Average Number of Trips per } \\
\text { Day }\end{array}$ & $3.8^{3}$ & 2.9 & 2.9 & 2.9 \\
\hline Urban** & & 3.0 & 3.0 & 3.0 \\
\hline Rural $^{\star *}$ & & 2.3 & 2.3 & 2.4 \\
\hline $\begin{array}{l}\text { Percent of Trips by Private } \\
\text { Vehicle }\end{array}$ & $83.4 \%^{3}$ & $79.4 \%$ & $80.5 \%$ & $81.2 \%$ \\
\hline Urban** & & $80.9 \%$ & $82.2 \%$ & $82.1 \%$ \\
\hline Rural $^{* *}$ & & $71.7 \%$ & $71.0 \%$ & $79.2 \%$ \\
\hline Average Trip Length (miles) & $9.7^{3}$ & 17.0 & 17.2 & 17.3 \\
\hline Urban** & & 16.2 & 16.7 & 16.6 \\
\hline Rural** $^{* *}$ & & 21.1 & 20.3 & 21.0 \\
\hline
\end{tabular}

* Car Access for U.S. Population defined as Households with Access to 1 or More Vehicles

** Residence in Urban Area for AMT Participants defined as those with two or more daily destinations within an urban area as defined by the U.S. Census in 2015

${ }^{1}$ Year 2015 Data from U.S. Census Bureau (2015), 2015 American Community Survey 1-Year Estimates

2 Year 2014 Data from Pew Research Center (2015), Car, bike or motorcycle? Depends on where you live

${ }^{3}$ Year 2009 Data from Department of Transportation (2015), Passenger Facts and Figures 2015 


\section{0 psiTurk Platform Specifications}

psiTurk (psiTurk 2014) is an open source platform for managing Amazon Mechanical Turk HITs and interactions with workers. Functionality includes the ability to issue and expire HITs, restrict an experiment to workers who live inside the United States or have not already taken a HIT, and track the duration of user interaction with the HIT. The PsiTurk application ran from its own cloud-based server hosted by Amazon Web Services and saved data within a MySQL database. This study constrained the number of open hits to 15 at any one time to reduce the load on this server. A m3.medium sized EC2 instance from AWS in place of a t2.small instance was also implemented to improve application reliability.

Implementation of the psiTurk application required users to be directed from a HIT hosted on a AMT web page to a separate browser window. From this intermediate psiTurk window, users were directed to another browser window hosting the Retrospective Mobility data collection app. Users then needed to interact with termination buttons in each of these windows to successfully register a completed HIT. Users were exposed to timeouts and other compatibility difficulties with browser and ad blocking software because of these numerous hand-offs. Future iterations of the platform will look to more seamlessly integrate AMT, psiTurk and the data collection application in order to simplify the user onboarding process and improve user experience. 